\title{
Some Remarks on Approximate Reasoning and Bandler-Kohout Subproduct
}

\author{
Katarzyna Miś(iD and Michał Baczyński ${ }^{(\otimes)}$ (iD \\ Faculty of Science and Technology, University of Silesia in Katowice, \\ Bankowa 14, 40-007 Katowice, Poland \\ \{katarzyna.mis, michal . baczynski\}@us.edu.pl
}

\begin{abstract}
In our contribution we give some remarks and conclusions regarding reasoning schemas used in approximate reasoning. Based on created computer tool for image customization we give some advices regarding FITA. Also, we show some facts regarding Bandler-Kohout subproduct and we present results for several inference schemas.
\end{abstract}

Keywords: Bandler-Kohout subproduct $\cdot$ Compositional rule of inference $\cdot$ Fuzzy implications $\cdot$ Fuzzy connectives

\section{Introduction}

Approximate reasoning is an often used concept when it is required to obtain meaningful results from imprecise data. One idea comes from Zadeh [15], where we use some inference schemas to receive such results. These schemas are based on their classic versions like:

- hypothetical syllogism

$$
\frac{A \rightarrow B \wedge B \rightarrow C}{\therefore \quad A \rightarrow C}
$$

- modus ponens

$$
\frac{A \rightarrow B \wedge A}{\therefore \quad B}
$$

- modus tollens

$$
\frac{A \rightarrow B \wedge \neg B}{\therefore \quad \neg A}
$$

- reduction to absurdity

$$
\frac{\neg A \rightarrow B \wedge \neg B}{\therefore \quad A}
$$

where $A, B, C$ are any statements. The one which is the most commonly applied is generalized modus ponens (GMP): 
RULE: $\quad$ IF $x$ is $A$, THEN $y$ is $B$.

FACT: $\quad x$ is $A^{\prime}$.

CONCLUSION: $\quad y$ is $B^{\prime}$,

where $x, y$ are some objects and $A, B, A^{\prime}, B^{\prime}$ are some properties. In our case these properties are represented by fuzzy sets so we are able to compute the values of $B^{\prime}$ using the following method:

$$
B^{\prime}:=A^{\prime} \star R,
$$

where $R$ is a fuzzy relation and $\star$ is a composition of such $R$ and a fuzzy set $A^{\prime}$. More precisely, if we use the composition proposed by Zadeh - the compositional rule of inference (CRI), which is the most popular, we have the following formula for our result:

$$
B^{\prime}(y):=\sup _{x \in X} T\left(A^{\prime}(x), I(A(x), B(y))\right), \quad y \in Y,
$$

where $T$ is a t-norm (or any generalization of classical conjunction) and $I$ is a fuzzy implication (or any generalization of classical implication). Another kind of a composition $\star$ is the Bandler-Kohout subproduct (BKS) $[3,13]$. In this case we usually use different model of a relation $R$ and we consider the following formula:

$$
B^{\prime}(y):=\inf _{x \in X} I\left(A^{\prime}(x), T(A(x), B(y))\right), \quad y \in Y .
$$

For both of them we almost always require a minimal property of interpolativity. It is nothing else but satisfying a classic version of modus ponens, i.e., if we take $A^{\prime}=A$, then we should obtain $B^{\prime}=B$. Therefore, if we take into account all possible values of fuzzy sets $A, B$ (i.e., the unit interval $[0,1]$ ), then from (1) and (2) we receive the following two functional equations:

$$
\begin{array}{ll}
y=\sup _{x \in[0,1]} T(x, I(x, y)), & y \in[0,1], \\
y=\inf _{x \in[0,1]} I(x, T(x, y)), & y \in[0,1],
\end{array}
$$

where $T$ is a t-norm and $I$ is a fuzzy implication. Both functional equations (or their counterparts written as inequalities) were studied extensively in the literature for different classes of operations (see $[2,14]$ ).

In many applications of generalized modus ponens, the rule base is not consisted of only one rule IF - THEN, but is contains more of them. Hence we have the following scheme:

$$
\begin{aligned}
& \text { IF } x \text { is } A_{1} \text {, THEN } y \text { is } B_{1} \text {, } \\
& \text { IF } x \text { is } A_{2} \text {, THEN } y \text { is } B_{2} \text {, } \\
& \ldots \\
& \text { IF } x \text { is } A_{n} \text {, THEN } y \text { is } B_{n} \text {, } \\
& \text { FACT: } x \text { is } A^{\prime} .
\end{aligned}
$$


To obtain the conclusion: $y$ is $B^{\prime}$ (which is based on all given rules) there are two main strategies (see $[2,10])$ :

- FATI - First Aggregate, Then Infer:

$$
\text { Aggregate: } \begin{aligned}
R(x, y) & =G\left(R_{1}(x, y), \ldots, R_{n}(x, y)\right) \\
& =G\left(I\left(A_{1}(x), B_{1}(y)\right), \ldots, I\left(A_{n}(x), B_{n}(y)\right)\right), \\
\text { Infer: } B^{\prime}(y): & =A^{\prime}(x) \circ R(x, y)
\end{aligned}
$$

- FITA - First Infer, Then Aggregate:

$$
\text { Infer: } \begin{aligned}
B_{i}^{\prime}(y)=A^{\prime}(x) \stackrel{T}{\circ} R_{i}(x, y)= & A^{\prime}(x) \stackrel{T}{\circ} I\left(A_{i}(x), B_{i}(y)\right), \\
& \text { for all } i \in\{1, \ldots, n\},
\end{aligned}
$$

Aggregate: $B^{\prime}(y)=G\left(B_{1}^{\prime}(y), \ldots, B_{n}^{\prime}(y)\right)$,

where $G$ is some aggregation function (it can be a t-norm).

Later we will present some conclusions regarding usage of FITA. Moreover, in our paper we would like to present some other results and corollaries that we obtained during work on a computer tool for image customization. We prepared it in order to test if our theoretical results concerning some reasoning schemas have an impact on applications of approximate reasoning (and what type). We used GMP scheme and this is a starting point for some other investigations.

The paper is organized as follows. Section 2 contains briefly description of the mentioned tool and some preliminaries which will be used in a sequel. In Sect. 3 we present some observations based on exemplary images. In Sect. 4 we conclude our paper and give some plans for future work.

\section{Basic Notions and Facts}

\subsection{Preliminaries}

To make this paper self-contained we placed here some basic definitions regarding fuzzy connectives and some aggregation operators.

Definition 2.1 (see $[2,9]$ ). A non-increasing function $N:[0,1] \rightarrow[0,1]$ is called a fuzzy negation, if $N(0)=1, N(1)=0$. Moreover, a fuzzy negation $N$ is called

(i) strict if it is strictly decreasing and continuous,

(ii) strong if it is an involution, i.e., $N(N(x))=x$ for all $x \in[0,1]$.

Definition $2.2([8])$. Let $n \in \mathbb{N}$. An aggregation function in $[0,1]^{n}$ is a function $A^{(n)}:[0,1]^{n} \rightarrow[0,1]$ which satisfies the following conditions:

(i) it is nondecreasing (in each variable),

(ii) $A^{(n)}(0, \ldots, 0)=0$ and $A^{(n)}(1, \ldots, 1)=1$. 
Definition 2.3 ([7]). A function $S:[0,1]^{2} \rightarrow[0,1]$ is called a semicopula if it satisfies the following conditions:

(i) $S(x, 1)=S(1, x)=x, \quad x \in[0,1]$,

(ii) $S$ is non-decreasing with respect to each variable.

Definition 2.4 ([8]). An n-ary mean in $[0,1]^{n}$ is an aggregation function $M^{(n)}$ which is internal, i.e.,

$$
\min \left\{x_{1}, \ldots, x_{n}\right\} \leq M^{(n)}\left(x_{1}, \ldots, x_{n}\right) \leq \max \left\{x_{1}, \ldots, x_{n}\right\},
$$

for all $\left(x_{1}, \ldots, x_{n}\right) \in[0,1]^{n}$.

Definition 2.5 ([9, Definition 1.1]). A function $T:[0,1]^{2} \rightarrow[0,1]$ is called a triangular norm (shortly t-norm) if it satisfies the following conditions, for all $x, y, z \in[0,1]:$

(T1) $T(x, y)=T(y, x)$, i.e., $T$ is commutative,

(T2) $T(x, T(y, z))=T(T(x, y), z)$, i.e., $T$ is associative,

(T3) $] T$ is non-decreasing with respect to each variable,

(T4) $T(x, 1)=x$.

Definition 2.6 ([1]). The diagonal of a t-norm $T$ is the function $\delta_{T}:[0,1] \rightarrow$ $[0,1]$ defined by

$$
\delta_{T}(x)=T(x, x), \quad x \in[0,1] .
$$

For more detailed facts about diagonals of t-norms see [11].

Definition 2.7 ([8, Definition 2.45]). A function $F:[0,1]^{n} \rightarrow \overline{\mathbb{R}}$ is idempotizable if its diagonal $\delta_{F}$ is strictly increasing and satisfies $\operatorname{ran}\left(\delta_{F}\right)=\operatorname{ran}(F)$.

Definition 2.8 ([8, Definition 4.2]). A function $M:[0,1]^{n} \rightarrow[0,1]$ is an average in $[0,1]^{n}$ if there exists a nondecreasing and idempotizable function $F:[0,1]^{n} \rightarrow \mathbb{R}$ such that

$$
F=\delta_{F} \circ M
$$

In this case, we say that $M$ is as average associated with $F$ in $[0,1]^{n}$.

Definition 2.9 ([2, Definition 1.1.1]). A function $I:[0,1]^{2} \rightarrow[0,1]$ is called a fuzzy implication if it satisfies the following conditions:

(I1) I is non-increasing with respect to the first variable,

(I2) I is non-decreasing with respect to the second variable,

(I3) $I(0,0)=I(1,1)=1$ and $I(1,0)=0$.

The set of all fuzzy implications will be denoted by $\mathcal{F} \mathcal{I}$.

Definition 2.10 (see [2]). We say that a fuzzy implication I satisfies

(i) the left neutrality property, if

$$
I(1, y)=y, \quad y \in[0,1]
$$


(ii) the ordering property, if

$$
x \leq y \Longleftrightarrow I(x, y)=1, \quad x, y \in[0,1] .
$$

Definition 2.11 ([2, Definition 2.5.1]). A function $I:[0,1]^{2} \rightarrow[0,1]$ is called an $R$-implication if there exists a t-norm $T$ such that

$$
I(x, y)=\sup \{t \in[0,1]: T(x, t) \leq y\}, \quad x, y \in[0,1] .
$$

If $I$ is generated from a $t$-norm $T$, then it will be denoted by $I_{T}$.

Definition 2.12 ([2, Definition 1.4.15]). Let I be a fuzzy implication. A function $N_{I}:[0,1] \rightarrow[0,1]$ given by

$$
N_{I}(x)=I(x, 0), \quad x \in[0,1],
$$

is called the natural negation of $I$.

Example 2.13. Some basic t-norms and generated R-implications are presented in Fig. 1.

\begin{tabular}{|c|c|}
\hline t-norm $T$ & R-implication $I_{T}$ \\
\hline \hline$T_{\mathbf{D}}(x, y)= \begin{cases}0, & x, y \in[0,1) \\
\min \{x, y\}, & \text { otherwise }\end{cases}$ & $I_{\mathbf{W B}}(x, y)= \begin{cases}1, & x<1 \\
y, & x=1\end{cases}$ \\
\hline$T_{\mathbf{L K}}(x, y)=\max \{0, x+y-1\}$ & $I_{\mathbf{L K}}(x, y)=\min \{1,1-x+y\}$ \\
\hline$T_{\mathbf{P}}(x, y)=x \cdot y$ & $I_{\mathbf{G G}}(x, y)= \begin{cases}1, & x \leq y \\
\frac{y}{x}, & x>y\end{cases}$ \\
\hline$T_{\mathbf{M}}(x, y)=\min \{x, y\}$ & $I_{\mathbf{G D}}(x, y)= \begin{cases}1, & x \leq y \\
y, & x>y\end{cases}$ \\
\hline
\end{tabular}

Fig. 1. Examples of basic R-implications.

\subsection{Description of a Computer Tool}

Let us take any picture where colors are given in RGB romat. Any pixel of such image can be seen as an object where we could adjust properties according to user choice/preferences. For instance, the user wants to change the color palette like below:

$$
[76,153,0] \rightarrow[128,255,0]
$$

from green to lighter between yellow and green $[255,255,0] \rightarrow[255,178,102]$

from yellow to one with orange tones. 
We can use GMP to compute values of such "vectors" of all pixels.

Let us consider some examples. Firstly, let us take the pair $\left(T_{\mathbf{L K}}, I_{\mathbf{L K}}\right)$. In the phase of aggregating (here for FITA) we need to apply an aggregation function. It turns out the same t-norm will not give satisfying results. Let us compare images presented in Fig. 2. Better results (according to our subjective opinion) are obtained in Fig. 2(b) and worse are presented in Fig. 2(c). For this pair we used different aggregation methods - the arithmetic mean and the Lukasiewicz t-norm. In the next part of our paper we give some possible justification which shows why it is better to apply some means instead of t-norms or other class of aggregation functions.

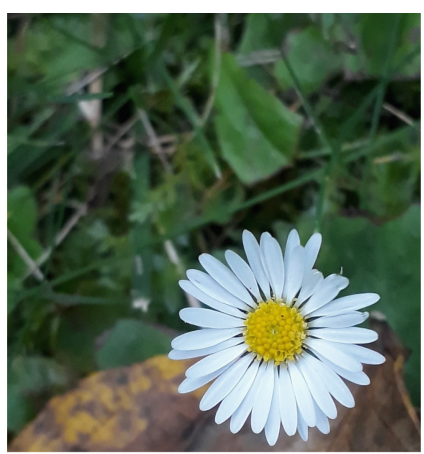

(a) The original image

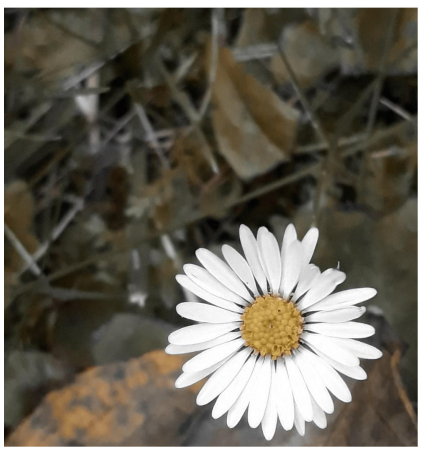

(b) Image obtained with $\left(T_{\mathbf{L K}}, I_{\mathbf{L K}}\right)$ and arithmetic mean for aggregating

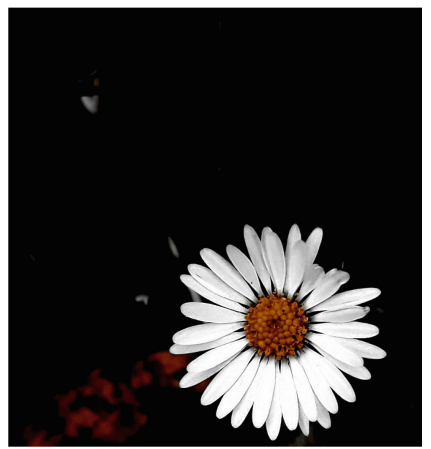

(c) Image obtained with $\left(T_{\mathbf{L K}}, I_{\mathbf{L K}}\right)$ and $T_{\mathbf{L K}}$ for aggregating

Fig. 2. Examples of images used in our programme - CRI. 


\section{Main Results}

\subsection{Case of FITA}

As we mentioned in Introduction, the basic property for compositions like CRI or BKS is interpolativity. Sometimes it is also called consistency [5] (in fact in $[4,5]$ the authors showed that FATI is not consistent). However, when we are thinking about this property we focus mainly on IF - THEN rules. Nevertheless, for the cases of more than one rule it is important to choose a proper aggregation operator. Note that for the rule base (3) and the fact $x$ is $A^{\prime}$ and taking $A^{\prime}=A_{i}, i \in\{1, \ldots, n\}$, we can check interpolativity separately for each rule. So for the aggregation operator $G$ we would obtain $G\left(B_{1}, \ldots, B_{n}\right)$. Based on intepolativity, an intuitive approach is when $B_{i}=B, i \in\{1, \ldots, n\}$ and then $G(B, \ldots, B)$ should be equal to $B$, which means $G$ is idempotent. Such functions are for instance OWA functions, medians, averaging aggregation functions. Among them there are also means.

Remark 3.1 ( $c f[8]$ ). For many t-norms and means there is the following relationship between $M$ (a mean) and $T$ (a t-norm):

$$
\delta_{T} \circ M=T .
$$

Example 3.2. Some classical means associated with t-norms are presented in Fig. 3.

\begin{tabular}{|c|c|c|}
\hline$M$ & $T$ & $\delta_{T}$ \\
\hline \hline$T_{\mathbf{M}}$ & $T_{\mathbf{M}}$ & $x$ \\
\hline$\frac{x+y}{2}$ & $T_{\mathbf{L K}}$ & $\max \{0,2 x-1\}$ \\
\hline$\sqrt{x y}$ & $T_{\mathbf{P}}$ & $x^{2}$ \\
\hline$\frac{2}{\frac{1}{x}+\frac{1}{y}}$ & $T_{\mathbf{H}}(x, y)= \begin{cases}0, & x=0 \wedge y=0, \\
\frac{x y}{x+y-x y}, & \text { otherwise. }\end{cases}$ & $\frac{x^{2}}{2 x-x^{2}}$ \\
\hline
\end{tabular}

Fig. 3. Classical means, corresponding t-norms and their diagonals.

Motivated by the above usage of means and t-norms we can define the following class of aggregation functions.

Proposition 3.3. Let $G:[0,1]^{2} \rightarrow[0,1]$ be an idempotent function and let $T_{1}, T_{2}$ be $t$-norms. Then a function $G_{T_{1}, T_{2}}:[0,1]^{2} \rightarrow[0,1]$ defined by a formula

$$
G_{T_{1}, T_{2}}(x, y)=G\left(T_{1}(x, y), T_{2}(x, y)\right), \quad x, y \in[0,1]
$$

is a commutative semicopula. 
Proof. It is easy to see that all properties are satisfied. For instance an existence of the neutral element 1 is as follows:

$$
G_{T_{1}, T 2}(1, y)=G\left(T_{1}(1, y), T_{2}(1, y)\right)=G(y, y)=y, \quad y \in[0,1]
$$

Proposition 3.4. Let $G, T_{1}, T_{2}:[0,1]^{2} \rightarrow[0,1]$, let $G$ be an idempotent and let $T_{1}, T_{2}$ be t-norms. If $T_{1}=T_{2}$ then a function $G_{T_{1}, T_{2}}$ defined by a formula (6) is a t-norm.

Proof. It is clear since for $T_{1}=T_{2}$ and $x, y \in[0,1]$ we have

$$
G_{T_{1}, T_{2}}(x, y)=G\left(T_{2}(x, y), T_{2}(x, y)\right)=T_{2}(x, y) .
$$

However usually $G_{T_{1}, T_{2}}$ is not a t-norm.

Example 3.5. Let $G$ be the arithmetic mean and $T_{1}=T_{\mathbf{M}}, T_{2}=T_{\mathbf{L K}}$. Then for $x=0.8, y=0.8$ and $z=0.5$ we have

$$
\begin{aligned}
G_{T_{\mathbf{M}}, T_{\mathbf{L K}}}\left(G_{T_{\mathbf{M}}, T_{\mathbf{L K}}}(x, y), z\right) & =G_{T_{\mathbf{M}}, T_{\mathbf{L K}}}(G(0.8,0.6), 0.5) \\
& =G_{T_{\mathbf{M}}, T_{\mathbf{L K}}}(0.7,0.5)=G(0.5,0.2)=0.35,
\end{aligned}
$$

while

$$
\begin{aligned}
G_{T_{\mathbf{M}}, T_{\mathbf{L K}}}\left(x, G_{T_{\mathbf{M}}, T_{\mathbf{L K}}}(y, z)\right) & =G_{T_{\mathbf{M}}, T_{\mathbf{L K}}}(0.8, G(0.5,0.3)) \\
& =G_{T_{\mathbf{M}}, T_{\mathbf{L K}}}(0.8,0.4)=G(0.4,0.2)=0.3 .
\end{aligned}
$$

\subsection{Case of Usage BK-subproduct}

Firstly we cite the following result, which justify the reason of choice of pairs of functions for the generalized hypothetical syllogism and CRI.

Theorem 3.6 ([12, Theorem 3.2]). Let $T$ be a t-norm. Then the following statements are equivalent:

(i) $T$ is left-continuous.

(ii) The pair $\left(T, I_{T}\right)$ satisfies the following functional equation

$$
\sup _{z \in[0,1]}(T(I(x, z), I(z, y)))=I(x, y), \quad x, y \in[0,1] .
$$

In fact, we have the similar result for (CRI-GMP).

Proposition 3.7 For a t-norm $T$ the following statements are equivalent:

(i) $T$ is left-continuous.

(ii) The pair $\left(T, I_{T}\right)$ satisfies (CRI-GMP). 
Proof $(i) \Longrightarrow($ ii $)$ If $T$ is left continuous, then from Theorem 3.6 it satisfies (CRI-GHS). Putting $x=1$ in (CRI-GHS) we obtain

$$
\sup _{z \in[0,1]}(T(I(1, z), I(z, y)))=I(1, y), \quad y \in[0,1] .
$$

But $I_{T}$ satisfies the left neutrality property (NP) (see [2, Theorem 2.5.4]), so

$$
\sup _{z \in[0,1]}(T(z, I(z, y)))=y, \quad y \in[0,1]
$$

and thus (ii) is satisfied.

$($ ii $) \Longrightarrow(i)$ Let $T$ be a t-norm and let us assume that the pair $\left(T, I_{T}\right)$ satisfies (CRI-GMP), but $T$ is not left-continuous. Based on [2, Proposition 2.5.5] we know that residual principle is not valid, so there exist $x, y, z \in[0,1]$ such that $I_{T}(x, y) \geq z$ and $T(x, z)>y$. Then, using the fact that (CRI-GMP) is valid for these $x, y, z$, we have

$$
y<T(x, z) \leq T\left(x, I_{T}(x, y)\right) \leq \sup _{t \in[0,1]} T\left(t, I_{T}(t, y)\right)=y
$$

a contradiction.

Therefore, we know which pairs can be used in (CRI-GMP), like we considered earlier the pair $\left(T_{\mathbf{L K}}, I_{\mathbf{L K}}\right)$. Moreover, note that if we use it we don't have to take care about the commutativity, i.e., the same result we would obtain for the functional equation

$$
y=\sup _{x \in[0,1]} T\left(I_{T}(x, y), x\right), \quad y \in[0,1] .
$$

However, if we consider BK-subproduct where there is a different composition and a different relation $R$, then the situation is unlike. Usually, we consider the Eq. (BK-GMP). Nevertheless, when in case of CRI we can change the order of arguments, here we would obtain:

$$
y=\inf _{x \in[0,1]} I(T(x, y), x), \quad y \in[0,1]
$$

Of course, it makes a big difference since we have a fuzzy implication $I$. This is in fact so called supercomposition $\triangleright$ or actually a superdirect image of a fuzzy set $A$ under a relation $R[6]$ :

$$
R_{\triangleright}(A)(y):=\inf _{x \in X} I(R(x, y), A(x)), \quad y \in Y .
$$

Now, let us mention about another functional equation obtained from the generalized hypothetical syllogism and based on the Bandler-Kohout subproduct:

$$
I_{2}(x, y)=\inf _{z \in[0,1]} I_{1}(C(x, z), C(z, y)), \quad x, y \in[0,1]
$$


which is a generalization of the following equation, where $A, B, C$ are fuzzy sets,

$$
I(A(x), B(y))=\inf _{z \in Z} I(T(A(x), C(z)), T(C(z), B(y))), \quad x \in X, y \in Y .
$$

The next two results can be proven as corollaries from our previous article [12].

Proposition 3.8. Let $I \in \mathcal{F} \mathcal{I}$ satisfies (NP) and let $C$ be a semicopula. If the triplet $(C, I, I)$ satisfies (BK-GHS), then the pair $(C, I)$ satisfies (BK-GMP).

Proposition 3.9. For any $t$-norm $T$ the pair $\left(T, I_{T}\right)$ satisfies (BK-GMP).

However, in applications except interpolativity property we know that we have discrete cases, so the infimum is in fact the minimum. Moreover, these results are also valid for such cases.

Proposition 3.10. Let $A, B, C$ be fuzzy sets and let $T$ be a t-norm. If $C$ is normal, then the triplet $\left(T, I_{T}, I_{T}\right)$ satisfies $(7)$.

Proposition 3.11. Let $A, B$ be fuzzy sets and let $T$ be a $t$-norm. If $A$ is normal, then the pair $\left(T, I_{T}\right)$ satisfies

$$
B(y)=\inf _{x \in X} I(A(x), T(A(x), B(y))), \quad y \in Y .
$$

Note that for any fuzzy implication $I$ with (OP) we have the following equality $T(x, y) \leq x \Longleftrightarrow I(T(x, y), x)=1, x, y \in[0,1]$, and then $I(x, T(x, y))<1$. Hence, for such fuzzy implications $I$ Eq. (BK-GMP-Rev) does not have any solutions. But as we can see on Fig. 4, usage of such order gave in our tool results which cannot be called useless. This could be a reason why we should consider these composition (BKS and supercomposition $\triangleright$ ) in different frameworks then $\mathrm{CRI}$ and check some other properties besides interpolativity.

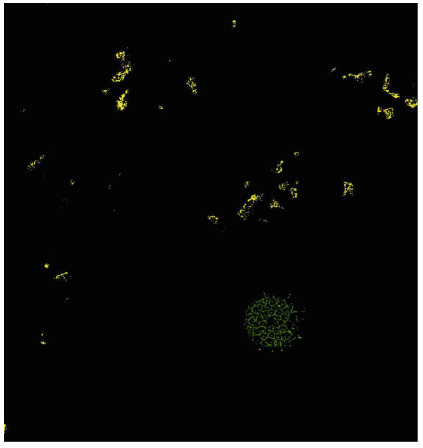

(a) Image obtained from BKS with $\left(T_{\mathbf{P}}, I_{\mathbf{G G}}\right)$

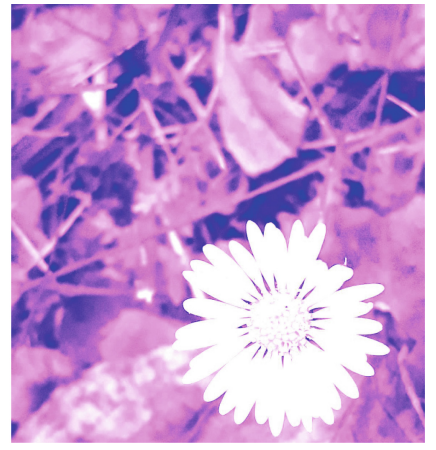

(b) Image obtained with supercomposition $\triangleright\left(T_{\mathbf{P}}, I_{\mathbf{G G}}\right)$

Fig. 4. Examples of images used in our programme - BK-subproduct. 
Now let us give some other examples of using BK-subproduct for other reasoning schemas which does and does not have solutions. Firstly, let us consider generalized modus tollens:

$$
N(x)=\inf _{y \in[0,1]} I(N(y), T(x, y)), \quad x \in[0,1] .
$$

Remark 3.12. Note that (BK-GMT) have no solutions. Indeed,

$$
\inf _{y \in[0,1]} I(N(y), T(x, y)) \leq I(N(0), T(x, 0))=I(1,0)=0 \neq N(x),
$$

for all $x \in[0,1]$.

Hence, now let us focus on the following functional equation

$$
N_{1}(x)=\inf _{y \in[0,1]} I\left(T(x, y), N_{2}(y)\right), \quad x \in[0,1],
$$

(BK-GMT-Rev)

where $I \in \mathcal{F} \mathcal{I}, T$ is a t-norm and $N_{1}, N_{2}$ are some fuzzy negations.

Proposition 3.13. Let $I \in \mathcal{F} \mathcal{I}, T$ be a $t$-norm and $N_{1}, N_{2}$ be any fuzzy negations. Then the following statements are equivalent:

(i) The quadruple $\left(T, I, N_{1}, N_{2}\right)$ satisfies (BK-GMT-Rev).

(ii) $N_{1}=N_{I}$.

Proof. It is enough to see that

$$
\inf _{y \in[0,1]} I\left(T(x, y), N_{2}(y)\right)=I(T(x, 1), N(1))=I(x, 0)=N_{I}(x) .
$$

Next, let us consider the functional equation obtained from the reduction to absurdity:

$$
x=\inf _{y \in[0,1]} I(N(y), T(N(x), y)), \quad x \in[0,1] .
$$

Here note that there is an analogous case as in (BK-GMT) equation.

Remark 3.14. Let $I \in \mathcal{F} \mathcal{I}, T$ be a t-norm and $N$ be a fuzzy negation. Then there does not exist any triplet $(T, I, N)$ that satisfies (BK-GRA). Indeed, let us take $x \in[0,1]$. Then

$$
\inf _{y \in[0,1]} I(N(y), T(N(x), y)) \leq I(N(0), T(N(x), 0))=I(1,0)=0 \neq x
$$

for any $x>0$. 
Hence, similarly as for (BK-GMT), when we consider the reverse order of arguments we can write the following functional equation:

$$
x=\inf _{y \in[0,1]} I(T(N(x), y), N(y)), \quad x \in[0,1] .
$$

Proposition 3.15. Let $I \in \mathcal{F} \mathcal{I}, T$ be a t-norm, $N$ be a negation and let $N_{I}$ be a strict negation. Then the following statements are equivalent:

(i) The triplet $(T, I, N)$ satisfies (BK-GRA-Rev).

(ii) $N_{I}^{-1}=N$.

Proof. Take $x \in[0,1]$. Then

$$
\inf _{y \in[0,1]} I(T(N(x), y), N(y))=I(T(N(x), 1), N(1))=I(N(x), 0),
$$

so from the fact $x$ is arbitrarily fixed we have

$$
x=N_{I}(N(x)) \Longleftrightarrow N_{I}^{-1}(x)=N(x),
$$

because $N_{I}$ is strict.

\section{Conclusions}

In this paper we briefly described the tool which we created. We can conclude that in case of FITA it may be worth to use means associated with t-norms for the aggregation part. Also, we noticed that a different look on BK-subproduct may give various results. Moreover, we presented some results regarding different functional equations. For the future work we would like to examine BKsubproduct deeply and investigate differences, which we discovered from our tool, between it and CRI.

Acknowledgment. The work on this paper was supported by the National Science Centre, Poland, under Grant No. 2015/19/B/ST6/03259.

\section{References}

1. Alsina, C., Frank, M., Schweizer, B.: Associative Functions: Triangular Norms and Copulas. World Scientific Publishing, Singapore (2006). https://doi.org/10.1142/ 6036

2. Baczyński, M., Jayaram, B.: Fuzzy Implications. In: Studies in Fuzziness and Soft Computing, vol. 231, Springer, Heidelberg (2008). https://doi.org/10.1007/978-3540-69082-5

3. Bandler, W., Kohout, L.: Semantics of implication operators and fuzzy relational products. Int. J. Man-Mach. Stud. 12(1), 89-116 (1980). https://doi.org/10.1016/ S0020-7373(80)80055-1 
4. Buckley, J.J.: Erratum to "Can approximate reasoning be consistent?": [Fuzzy sets and systems 65 (1994) 13-18]. Fuzzy Sets Syst. 103(1), 189-190 (1999). https:// doi.org/10.1016/S0165-0114(98)00443-6

5. Buckley, J.J., Hayashi, Y.: Can approximate reasoning be consistent? Fuzzy Sets Syst. 65(1), 13-18 (1994). https://doi.org/10.1016/0165-0114(94)90243-7

6. De Baets, B.: Analytical solution methods for fuzzy relational equations. In: Dubois, D., Prade, H. (eds.) Fundamentals of Fuzzy Sets. FSHS, vol. 7, pp. 291340. Springer, Boston (2000). https://doi.org/10.1007/978-1-4615-4429-6_7

7. Durante, F., Sempi, C.: Semicopulae. Kybernetika 41(3), 315-328 (2005)

8. Grabisch, M., Marichal, J., Mesiar, R., Pap, E.: Aggregation Functions (Encyclopedia of Mathematics and its Applications). Cambridge University Press, Cambridge (2009). https://doi.org/10.1017/CBO9781139644150

9. Klement, E.P., Mesiar, R., Pap, E.: Triangular Norms. Kluwer Academic Publishers, Dordrecht (2000). https://doi.org/10.1007/978-94-015-9540-7

10. Klir, G.J., Bo, Y.: Fuzzy Sets and Fuzzy Logic Theory and Applications. Prentice Hall, New Jersey (1995)

11. Mesiar, R., Navara, M.: Diagonals of continuous triangular norms. Fuzzy Sets Syst. 104(1), 35-41 (1999). https://doi.org/10.1016/S0165-0114(98)00256-5

12. Miś, K., Baczyński, M.: Different forms of generalized hypothetical syllogism with regard to R-implications. In: Rutkowski, L., Scherer, R., Korytkowski, M., Pedrycz, W., Tadeusiewicz, R., Zurada, J.M. (eds.) ICAISC 2019. LNCS (LNAI), vol. 11508, pp. 304-313. Springer, Cham (2019). https://doi.org/10.1007/978-3-030-20912$4 \_29$

13. Pedrycz, W.: Applications of fuzzy relational equations for methods of reasoning in presence of fuzzy data. Fuzzy Sets Syst. 16, 163-175 (1985). https://doi.org/ 10.1016/S0165-0114(85)80016-6

14. Trillas, E., Alsina, C., Pradera, A.: On MPT implication functions for fuzzy logic. Rev. R. Acad. Cien. Ser. A Mat. 98, 259-271 (2004)

15. Zadeh, L.A.: Outline of a new approach to the analysis of complex systems and decision processes. IEEE Trans. Syst. Man Cyber. 3, 28-44 (1973). https://doi. org/10.1109/TSMC.1973.5408575 